\section{KOMPASS}

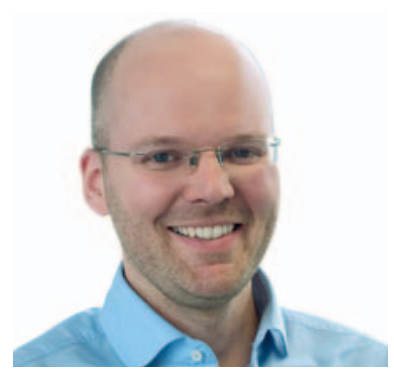

PD Dr. Sascha Gerdes

Klinik für Dermatologie, Venerologie und Allergologie

Universitätsklinikum Schleswig-Holstein, Campus Kiel

\title{
Wirkungen und Risiken von Biologika bei Psoriasis
}

\author{
Ausgewählt und kommentiert von \\ PD Dr. Sascha Gerdes (Kiel)
}

Psoriasis ist eine der häufigsten chronisch-entzündlichen Erkrankungen in der Dermatologie. Bis zum heutigen Tag sind insgesamt 12 verschiedene systemische Medikamente zur Behandlung von Psoriasis vulgaris zugelassen - 7 von diesen Medikamenten gehören zur Gruppe der Biologika. Die erste Generation von Biologika sind die TNF-a-Inhibitoren (Adalimumab, Etanercept und Infliximab), deren Patente zum Teil bereits ausgelaufen sind. Für Etanercept und Infliximab wurden die ersten Biosimilars zugelassen, wodurch die Preise für diese insgesamt teure Medikamentengruppe sukzessive gesunken sind. Damit bilden Biologika die Mehrzahl der zur Verfügung stehenden Präparate zur systemischen Therapie von Psoriasis vulgaris.

Die neueste Generation der Biologika besteht aus den Interleukin-17-Inhibitoren, die zuletzt durch Brodalumab ergänzt wurden, einem Antikörper der erstmals die Untereinheit A des IL-17-Rezeptors blockiert. Die Arbeit von Attia und Kollegen fasst die Ergebnisse des klinischen Studienprogramms mit insgesamt 4118 Patienten zusammen. Bemerkenswert ist die starke Wirksamkeit bei gleichzeitig geringer Anzahl von unerwünschten Arzneimittelwirkungen (UAW). Schlüsselt man die UAW nach einzelnen Ereignissen auf, so finden sich keine signifikanten Unterschiede zwischen den UAW der Behandlungsgruppen und den UAW der Placebogruppen. Aufgrund der noch neuen Entwicklung dieses Medikaments fehlen derzeit Langzeitbeobachtungen über mehrere Jahre.

Patienten legen allerdings besonderen Wert auf die Nachhaltigkeit eines Biologikums. Kromer und Kollegen konnten herausarbeiten, dass insbesondere Patienten, die in der Vortherapie mit konventionellen Systemtherapeutika oder Biologika behandelt wurden oder Patienten mit einer langen Krankheitsdauer eine hochwirksame Therapie mit einem guten Nutzen-Risiko-Profil und einem lang anhaltenden Behandlungserfolg wünschen.

Arnold und Kollegen konnten in ihrer Arbeit nachweisen, dass vor allem Ustekinumab eine hohe Therapietreue (drug survival) aufzeigt, gefolgt von den Anti-TNF-a-Biologika und den konventionellen Systemtherapeutika. In dieser Untersuchung fehlte die Gruppe der Biologika, die gegen Interleukin-17 gerichtet sind. Während konventionelle Systemtherapeutika und Infliximab am häufigsten wegen Nebenwirkungen abgesetzt wurden, konnte die Therapie mit den übrigen Biologika am häufigsten wegen eines therapeutischen Wirkversagens nicht fortgesetzt werden.

Biologika sind heute unverzichtbarer Bestandteil eines umfassenden Psoriasis-Managements. Insbesondere unter Berücksichtigung von Wirksamkeit, Sicherheit und Patientenpräferenzen haben sie einen hohen Stellenwert bei der Systemtherapie.

\section{KARGER}

() 2017 S. Karger GmbH, Freiburg

Fax +497614520714

information@karger.com

www.karger.com 
Attia A, Abushouk Al, Ahmed H, Gadelkarim M, Elgebaly A, Hassan Z, Abdel-Daim MM, Negida A: Safety and efficacy of brodalumab for moderate-to-severe plaque psoriasis:

A systematic review and meta-analysis. Clin Drug Investig 2017;37:439-451.

Background: Psoriasis is an inflammatory skin disease that affects $2-3 \%$ of the worldwide population. The interleukin-17 cytokine family has been proven to play a central role in the pathogenesis of psoriasis. Brodalumab is a novel biologic agent that targets interleukin-17 molecules and their receptors. We aimed to evaluate the safety and efficacy of brodalumab as a therapeutic agent for moderate-to-severe psoriasis in a meta-analysis framework.

Methods: A computer literature search of PubMed, OVID, Cochrane Central, EMBASE, EBSCO, Scopus, and Web of Science was conducted using relevant keywords. Data were extracted from eligible trials and analyzed using RevMan (Version 5.3 for windows) and OpenMeta [Analyst] software.

Results: Six clinical trials ( $\mathrm{n}=4118$ patients) were pooled in the final analysis. The pooled effect size favored brodalumab over placebo in terms of the Psoriasis Area and Severity Index (PASI) 75 [risk ratio $(\mathrm{RR})=12.61,95 \%$ confidence interval (CI) 9.74-16.34], PASI 90 ( $R R=28.72$, 95\% CI 18.34-44.98), and PASI $100(\mathrm{RR}=61.23,95 \% \mathrm{CI} 25.48-147.17)$. Analysis of secondary outcomes showed that brodalumab was superior to placebo in terms of static physician's global assessment $(\mathrm{RR}=32.53,95 \% \mathrm{CI} 13.80-76.69)$ and psoriasis symptoms inventory scores $(\mathrm{RR}=14.70,95 \% \mathrm{CI} 8.38-25.78)$. Meta-regression analysis found a significant linear association between the brodalumab dose and the effect size on PASI and psoriasis symptoms inventory scores. The rate of overall adverse events was slightly higher in the brodalumab group ( $R R=$ 1.13, 95\% CI 1.06-1.22); however, none of the individual adverse events were significantly higher in the brodalumab group, compared to the placebo group.

Conclusion: Brodalumab showed an acceptable safety profile and a robust efficacy in the treatment of moderate-to-severe plaque psoriasis. However, the current evidence is insufficient to confirm maintenance of these results in the long term; therefore, larger studies with longer follow-up periods are required.

Kromer C, Peitsch WK, Herr R, Schmieder A, Sonntag D, Schaarschmidt ML: Behandlungspräferenzen für Biologika bei Psoriasis: Erfahrene Patienten legen Wert auf Nachhaltigkeit. J Dtsch Dermatol Ges 2017;15:189-201.

Hintergrund und Ziele: Die Therapiezufriedenheit kann durch die Berücksichtigung von Patientenpräferenzen in der gemeinsamen Entscheidungsfindung verbessert werden. Kürzlich untersuchten wir Patientenpräferenzen für Eigenschaften von Biologika und fanden starke Präferenzen für Sicherheit und Wirksamkeit. Die vorliegende Studie hatte das Ziel, Auswirkungen von Therapieerfahrung auf diese Präferenzen zu erheben.

Patienten und Methoden: Präferenzen für Ergebnis- (Wahrscheinlichkeit einer 50\%igen und 90\%igen Verbesserung, Zeit bis zum Ansprechen, Nachhaltigkeit des Erfolgs, Wahrscheinlichkeit von leichten und schweren Nebenwir- kungen und Wahrscheinlichkeit eines ACR-20-Ansprechens) und Prozesseigenschaften (Behandlungsort, Behandlungshäufigkeit, Zeitaufwand und Applikationsweise) wurden bei 200 Teilnehmern mit mittelschwerer bis schwerer Psoriasis mit Hilfe von Conjoint-Analyse ermittelt. Der Einfluss aktueller und früherer Therapien, der Krankheitsdauer und der Behandlungszufriedenheit auf die «Relative Importance Scores» wurde durch Varianz-analysen, Post-hoc-Tests und multivariate Regressionen bestimmt.

Ergebnisse: Teilnehmer, die aktuell eine topische Therapie $(\mathrm{p}=0,02)$ oder eine Phototherapie $(\mathrm{p}=0,032)$ erhielten, hielten den Zeitaufwand der Behandlung für wichtiger als andere. Diejenigen, denen zuvor traditionelle Systemtherapien ( $\mathrm{p}=0,028)$ oder Biologika $(\mathrm{p}=0,044)$ verordnet worden waren, legten mehr Wert auf die Nachhaltigkeit als andere. Diese Eigenschaft gewann mit steigender Anzahl zuvor verabreichter systemischer Therapien $(p=0,045)$ und längerer Krankheitsdauer ( $p=0,018)$ an Bedeutung.

Fazit: Patientenpräferenzen für Biologika variieren abhängig von der Therapieerfahrung und Krankheitsdauer. Diese Aspekte sollten bei der gemeinsamen Entscheidungsfindung berücksichtigt werden.

\section{Arnold T, Schaarschmidt ML, Herr R, Fischer JE, Goerdt S,} Peitsch WK: «Drug-Survival» - Raten und Gründe für den Abbruch von Systemtherapien bei Psoriasis. J Dtsch Dermatol Ges 2016;14:1090-1101.

Hintergrund und Ziele: Mittelschwere bis schwere Psoriasis erfordert häufig eine langfristige systemische Behandlung. Die Therapietreue bezüglich eines Medikaments (Überlebensrate, «Drug Survival») reflektiert dessen Wirksamkeit, Sicherheit sowie die Zufriedenheit mit der Behandlung und ist ein Indikator für den Therapieerfolg. Das Ziel der vorliegenden Studie war die Ermittlung der «Drug-Survival»-Raten sowie der Gründe für den Abbruch einer Behandlung mit Fumarsäureestern (fumaric acid esters, FAE), Methotrexat (MTX), Acitretin (ACI), Cyclosporin A (CyA), Adalimumab (ADA), Etanercept (ETA), Infliximab (INF) und Ustekinumab (UST) bei Patienten mit mittelschwerer bis schwerer Psoriasis.

Patienten und Methoden: Wir führten eine retrospektive Analyse an $373 \mathrm{~Pa}$ tienten durch, die im Zeitraum 1/2003-5/2014 insgesamt 696 Behandlungen an einem deutschen Universitätsklinikum erhalten hatten.

Ergebnisse: Die unbereinigte Überlebenswahrscheinlichkeit war für UST am höchsten, gefolgt von ADA, ETA, INF, FAE, MTX, ACI und CyA. In der multivariaten Regressionsanalyse mit FAE als Referenz betrug die Hazard Ratio (HR) für einen Abbruch 0,14 (95\% Konfidenzintervall: 0,06-0,35) bei UST, $0,43(0,26-0,73)$ bei ADA, $2,11(1,14-3,91)$ bei ACI und 3,26 $(1,44-7,39)$ bei CyA. Das «Drug Survival» von INF war länger, wenn es in Kombination mit MTX eingesetzt wurde (HR 2,87; 1,21-6,81). Traditionelle systemische AntiPsoriatika sowie INF wurden am häufigsten aufgrund von Nebenwirkungen abgesetzt; alle anderen Biologika aufgrund ihrer Unwirksamkeit für kutane Psoriasis-Läsionen.

Schlussfolgerungen: «Drug-Survival»-Raten sollten bei der Therapieentscheidung berücksichtigt werden, um den Patienten eine bestmögliche Langzeitstrategie zu bieten. 